\title{
Regulation of 5-hydroxytryptamine synthesis
}

\section{By D. A. Bender, Courtauld Institute of Biochemistry, The Middlesex Hospital Medical School, London $W_{\mathrm{I}} P{ }_{7} P N$}

There is a great deal of evidence that changes of precursor availability, within the range of normal physiological variation, can affect the synthesis of neurotransmitters in the brain. The transmitter amine 5-hydroxytryptamine (serotonin; $5-\mathrm{HT}$ ) is derived from tryptophan, and it has been shown that changes in blood tryptophan levels are reflected in changes in brain 5 - HT synthesis. Since the blood tryptophan level is affected by the diet, it seems likely that food intake can affect 5HT synthesis in the brain.

The first step in $5-\mathrm{HT}$ synthesis is the hydroxylation of tryptophan to 5 hydroxytryptophan, catalysed by tryptophan hydroxylase (L-tryptophan monooxygenase; $E C$ I.14.I 6.4). This is followed by decarboxylation to the biologicallyactive amine, catalysed by aromatic amino acid decarboxylase (L-aromatic amino acid carboxy-lyase; $E C$ 4.1.1.28).

In the central nervous system, 5 -HT is stored in pre-synaptic vesicles before release as a neurotransmitter; thereafter it can either be taken up by the presynaptic neuron (where it may either be re-utilized or catabolized) or be catabolized in the post-synaptic neuron. The catabolism of 5-HT is again a twostep process; the first reaction is catalysed by monoamine oxidase (monoamine:oxygen oxidoreductase (deaminating); $E C$ 1.4.3.4) and yields 5-hydroxyindoleacetaldehyde as a transient intermediate, which is then oxidized to 5-hydroxyindoleacetic acid (5-HIAA), a reaction catalysed by aldehyde dehydrogenase (aldehyde:NAD oxidoreductase, $E C$ 1.2.1.3). 5-HIAA is cleared from the central nervous system and is excreted in the urine.

Control of the amount of $5-\mathrm{HT}$ in the central nervous system could conceivably occur by change in the rate of either synthesis or oxidative catabolism. Control of monoamine oxidase is not generally thought to be a physiological mechanism of $5^{-}$ HT regulation, although inhibition of the enzyme is frequently used clinically to increase brain amine levels (including $5-\mathrm{HT}$ ) in the treatment of psychological depression.

Aromatic amino acid decarboxylase is pyridoxal phosphate dependent, but appears to be relatively resistant to the effects of vitamin $B_{6}$ deficiency. Neither the total activity of the enzyme nor apo-enzyme:holoenzyme is altered in the brain or liver of animals severely-vitamin $\mathrm{B}_{6}$-deficient (Bayoumi \& Bender, unpublished observations). The enzyme has a low Michaelis constant $\left(K_{m}\right)$ for 5hydroxytryptophan (approximately $40 \mu \mathrm{M}$, Bender \& Coulson, 1972) and a relatively high activity in the brain. On these grounds it would appear unlikely that 
it is a regulatory enzyme in the synthesis of $5-\mathrm{HT}$. Furthermore, there is normally no detectable 5 -hydroxytryptophan in the brain, implying that this intermediate is rapidly decarboxylated. Under a variety of experimental conditions it has been shown that when brain tryptophan, 5- $\mathrm{HT}$ and 5-HIAA are increased (by the administration of tryptophan to rats) the concentration of 5-hydroxytryptophan in the brain remains below the limit of reliable detection (Eccleston, Ashcroft \& Crawford, 1965). Hence, it is extremely unlikely that decarboxylation is a regulatory step in 5 -HT synthesis.

$A$ priori, tryptophan hydroxylase would appear to be a logical point for regulation of $5-\mathrm{HT}$ synthesis; it is the first enzyme unique to the pathway. It is a two-enzyme system; the hydroxylase itself is a mixed-function oxidase, with tetrahydrobiopterin as cofactor. In the hydroxylation, the pterin is oxidized to dihydrobiopterin, and the second enzyme of the system is dihydrobiopterin reductase, a NADPH-dependent enzyme. Dihydrobiopterin reductase is common to tryptophan, phenylalanine and tyrosine hydroxylases; it is the hydroxylase component that differs in each instance. Unlike aromatic amino acid decarboxylase, which has a fairly wide distribution in the central nervous system, tryptophan hydroxylase is found only in serotoninergic neurons, and in the pineal gland, where 5 -HT is synthesized as an intermediate in the synthesis of melatonin (Kaufman, 1974).

In the presence of saturating amounts of tetrahydrobiopterin, tryptophan hydroxylase from rabbit brain has a $K_{m}$ for tryptophan of approximately $50 \mu \mathrm{M}$; when synthetic pterins are used, the $K_{m}$ for tryptophan can be very much higher (Kaufman (1974) quotes a value of $290 \mu \mathrm{M}$ using dimethyl tetrahydrobiopterin). Fernstrom \& Wurtman (1971) have estimated that the average brain concentration of tryptophan is approximately $30 \mu \mathrm{M}$ in the rat; other workers suggest a lower concentration, $5^{-25 \mu \mathrm{M}}$. These estimates are based on the known amount of tryptophan present per unit weight of the brain and the distribution volume available to tryptophan, and take no account of the possibility of locally higher concentrations as a result of anatomical compartmentation. Assuming that the average concentration of tryptophan in the brain approximates to the concentration of tryptophan available to the hydroxylase, this means that under normal physiological conditions the enzyme is less than half saturated with its substrate. Any increase in the concentration of tryptophan available to the enzyme will therefore result in an increase in the rate of hydroxylation of tryptophan to 5hydroxytryptophan, and presumably also an increase in the rate of $5-\mathrm{HT}$ synthesis. Eccleston et al. showed in a series of tryptophan-loading experiments that an increase in brain tryptophan was indeed reflected in an increase in the concentrations of both $5-\mathrm{HT}$ and 5 - $\mathrm{HIAA}$ in the brain. Tagliamonte and coworkers (Tagliamonte, Tagliamonte, Perez-Cruet \& Gessa, 1971) showed that a number of drugs that were known to increase brain 5 - $\mathrm{HT}$ concentration, other than by inhibition of monoamine oxidase, also acted to increase the brain tryptophan concentration. This was interpreted as evidence for the role of tryptophan availability in the regulation of $5-\mathrm{HT}$ synthesis. 
The availability of tryptophan in the brain for hydroxylation is governed by two factors, the uptake of tryptophan into the brain and the activity of competing pathways for tryptophan utilization.

There is some tryptophan oxygenase activity in the brain; this is probably indoleamine dioxygenase rather than tryptophan oxygenase (L-tryptophan:oxygen 2,3-oxidoreductase, decyclizing, EC I.I3.I I.II) (Hayaishi, r975). However, the activity of this enzyme is very low, and it is not likely to be very important in determining the fate of brain tryptophan. The major pathway of tryptophan utilization in the brain is protein synthesis. Tryptophan availability is unlikely to limit brain protein synthesis, since the $K_{m}$ of the rate-limiting enzyme of tryptophan utilization, the activating enzyme that esterifies the amino acid to tRNA, is extremely low; Liu, Chung \& Lee (1973) quote a value of $1-2.5 \mu \mathrm{M}$, depending on the assay conditions. The enzyme would therefore normally be saturated with substrate. However, changes in the rate of net protein synthesis would be expected to alter the distribution of the brain tryptophan pool, and hence its availability for hydroxylation.

The availability of tryptophan in the brain (for protein synthesis or hydroxylation and $5-\mathrm{HT}$ synthesis) is governed by uptake from the bloodstream. A number of studies have shown that alterations in circulating tryptophan are reflected in brain tryptophan and 5-HT concentrations. Eccleston et al. (1965) showed that administration of a relatively large dose of tryptophan to rats increased the circulating concentration of tryptophan and brain concentrations of tryptophan, 5-HT and 5-HIAA together. Green \& Curzon (1968) showed that reduction in circulating tryptophan after induction of liver tryptophan oxygenase by hydrocortisone administration to rats led to a decrease in brain $5-\mathrm{HT}$. Similarly, Cocchi and co-workers (Cocchi, di Giulio, Groppetti, Mantegazza, Müller \& Spano, 1975) showed that growth hormone administration to hypophysectomized rats led to a decrease in circulating tryptophan (as a result of increased protein synthesis, especially in the liver), and a decrease in brain tryptophan and 5-HIAA concentration. They also showed that in homozygous mutant dwarf mice, which lack growth hormone, the brain tryptophan and 5HIAA concentrations are higher than normal, as is the circulating plasma tryptophan concentration, and administration of growth hormone to these animals will reduce the concentrations of all three in parallel.

The availability of tryptophan in the brain is influenced by the active transport system of the blood-brain barrier; amino acids are not freely permeable into the brain, but are actively taken up by an enzyme system that is probably located in the walls of the cerebral blood vessels. A useful method for study of this transport system is the 'single-pass' perfusion technique of Oldendorf (1971), in which uptake of radioactive amino acid into the brain is measured after in vivo bolus infusion into the carotid artery. Such studies have extended earlier indications that there are several amino acid transport systems with different specificity; one has affinity for all of the so-called 'large neutral' amino acids, tryptophan, phenylalanine, tyrosine, leucine, isoleucine, valine and possibly also methionine and 
threonine (Pardridge \& Oldendorf, 1977). This common carrier mechanism means that these amino acids would all be expected to compete with one another for uptake into the brain, and therefore that changes in plasma tryptophan concentration might not be reflected in changes in brain tryptophan. Fernstrom and coworkers (Fernstrom, Madras, Munro \& Wurtman, I974) showed that this was so in a series of fasting and refeeding experiments. When animals were refed on a protein containing meal, although there was a considerable increase in plasma tryptophan, there was not the expected increase in brain tryptophan and $5-\mathrm{HT}$. They showed that this was associated with the concurrent increase in the plasma concentrations of the other 'large neutral' amino acids together with tryptophan, as a result of protein ingestion. They also showed that on refeeding the animals there was a good correlation between brain tryptophan concentration and tryptophan: 'large neutral' amino acids in plasma.

It is well established that, alone of the amino acids, plasma tryptophan is largely associated with albumin (McMenamy \& Oncley, 1958). Depending on the species, the nutritional status of the animal and the experimental technique used to assess the extent of albumin binding, only between $8-20 \%$ of plasma tryptophan is freely diffusible under normal conditions, with the remainder bound to albumin. Under conditions of physiological or pharmacological stress very much more can be freely diffusible. This albumin binding is a rapid equilibrium process; there is one binding site/molecule albumin, and in fasting human subjects Bender, Boulton \& Coulson (1975) estimated a dissociation constant of $32 \mu \mathrm{M}^{+1}$ using an equilibrium dialysis technique.

There is considerable controversy as to whether binding to albumin affects the availability of tryptophan for brain uptake, as any tryptophan taken into the brain (from the small diffusible pool) will be replaced rapidly from the larger pool of albumin-bound tryptophan (Yuwiler, Oldendorf, Geller \& Braun, I977). However, if competition between available tryptophan and other amino acids influences uptake into the brain, then not only will the ability to replenish the pool of diffusible tryptophan be important but also the size of that pool, since this, and not the total plasma tryptophan competes with the other amino acids for the brain uptake system. Hence, it is to be expected that factors that affect the binding of tryptophan to albumin will, by affecting the size of the plasma diffusibletryptophan pool, affect brain tryptophan uptake.

Many agents are known to displace tryptophan from albumin, including nonesterified fatty acids, which occur in plasma under physiological conditions (McMenamy \& Oncley, 1958; Curzon, Friedel \& Knott, 1973; Cunningham, Hay \& Stoner, 1975) and drugs such as salicylate (Smith \& Lakatos, I971). Tagliamonte and co-workers (Tagliamonte, Biggio, Vargiu \& Gessa, I973) showed that administration of salicylate to rats led to an increase in brain tryptophan and 5 - $\mathrm{HT}$ concentrations, despite a reduction in the total plasma tryptophan concentration. They attributed this to the displacement of tryptophan from albumin binding by the salicylate. Similarly, Knott \& Curzon (1972) and Curzon \& Knott (r974) showed that many physiological and drug-provoked changes in serum non- 
esterified fatty acids and diffusible tryptophan in rats were in the same direction, and increased diffusible tryptophan in plasma was associated with increased brain tryptophan, 5-HT and 5-HIAA. It would thus appear that in vivo the binding of tryptophan to albumin is an important determinant of tryptophan uptake into the brain.

In a number of studies with drugs, an increase in either the concentration of tryptophan in the brain or in the uptake of radioactive tryptophan into the brain over a short period of time has been shown to be related to the concentration of diffusible rather than total plasma tryptophan. Indeed, in many instances there has been an increase in the concentration of diffusible tryptophan, due to displacement from albumin binding, with a decrease in total plasma tryptophan, and an increase in brain tryptophan uptake (Tagliamonte et al. 1973). Studies with a variety of antischizophrenic phenothiazines indicate that both the serum diffusible tryptophan and the concentrations of competing amino acids, but apparently not the serum total tryptophan concentration, are determinants of brain tryptophan uptake (Bender \& Cockcroft, 1977).

Etienne, Young \& Sourkes (1976) have shown that the addition of albumin to the infusion bolus in 'single-pass' perfusion studies of brain tryptophan uptake reduced the uptake of tryptophan (but not of tyrosine, which does not bind to albumin). This would seem to be excellent evidence, obtained in vivo, and under relatively physiological conditions, for the importance of albumin binding as a regulatory factor. They did not observe as great an extent of inhibition of tryptophan transport in the presence of albumin as might be expected from its capacity to bind tryptophan, presumably because some of the bound tryptophan can replenish the pool of plasma diffusible tryptophan, even under the rapid conditions of 'single-pass' perfusion.

It thus appears to be possible to predict the relative rate of brain $5-\mathrm{HT}$ synthesis by measurement of the plasma concentrations of diffusible tryptophan and competing 'large neutral' amino acids. Several of the drug studies cited previously indicate that this is so. However, other factors are also involved in the regulation of brain 5 -HT synthesis. Hamon, Bourgoin \& Glowinski (1973) showed that there was inhibition of further 5 - $\mathrm{HT}$ synthesis by brain slices in vitro when 5 - $\mathrm{HT}$ was added to the incubation medium, and in vivo after administration of a monoamine oxidase inhibitor. There thus appears to be some kind of feedback regulation of 5 HT synthesis in the brain. After the administration of chloropromazine to rats, both brain tryptophan uptake and 5-HT accumulation are increased, but the increase in new $5-\mathrm{HT} /$ unit increase in tryptophan uptake is less in animals that have been treated with the drug chronically than in animals that have received a single dose (Bender, 1976). This indicates that the increased brain 5 -HT serves to limit further synthesis. It is not likely that this inhibition is simple product or endproduct inhibition of either enzyme; although such inhibition can be observed in vitro, it only occurs at concentrations several orders of magnitude greater than are likely to be encountered in vivo (Bender \& Coulson, 1972; Kaufman, 1974).

Shields \& Eccleston (1972) showed that electrical stimulation of the brain led to 
increased 5-HT synthesis, and Marsden \& Curzon (1976) showed that stimulation of the raphe nucleus of the brain led to increased brain $5-\mathrm{HT}$ synthesis, and an increased brain concentration of $5-\mathrm{HIAA}$, without changing the brain tryptophan concentration. Based on such observations, Mandell \& Knapp (1977) have suggested that there may be changes in the activity of tryptophan hydroxylase in the brain. It is not clear whether these activity changes involve changes in the amount of enzyme present, but Hamon and co-workers (Hamon, Burgoin, Héry, Ternaux \& Glowinski, 1976) have suggested that changes in the quaternary structure of the hydroxylase can modulate its activity. It must be assumed, albeit without evidence, that there is some kind of neuronal feedback to account for this regulation, since a direct metabolic effect seems unlikely.

The uptake of tryptophan across the blood-brain barrier is not the only factor governing the availability of tryptophan in serotoninergic neurons. Mandell \& Knapp (1977) have shown that there is a specific high affinity tryptophan transport system in the membrane of serotoninergic neurons. Certainly under some conditions this is likely to be a regulatory factor, since it has been shown to be affected by a number of factors that alter brain 5 -HT synthesis and synaptosomal uptake of tryptophan, without affecting the blood-brain barrier transport system.

In summary, it seems clear that a major factor in the regulation of brain $5-\mathrm{HT}$ synthesis, although not the only one, is the availability of tryptophan in the brain. Animal studies indicate that this can be estimated, to reasonable approximation, by measurement of plasma diffusible tryptophan and 'large neutral' amino acid concentrations. As well as this there are regulatory mechanisms which affect the formation of 5 -HT from the available tryptophan.

Finally, while a great deal of experimental evidence shows that drugs which affect 5-HT synthesis also alter behaviour, we know relatively little about the effects of physiological changes in the amount of 5 -HT in the brain, or in the rate of its synthesis and catabolism, on the neurotransmitter functions of $5-\mathrm{HT}$.

\section{REFERENCES}

Bender, D. A. (1976). Biachem. Pharmac. $25,1743$.

Bender, D. A., Boulton, A. P. \& Coulson, W. F. (1975). Biochem. Sac. Trans. 3, 193.

Bender, D. A. \& Cockcroft, P. M. (1977). Biochem. Soc. Trans. 5, 155.

Bender, D. A. \& Coulson, W. F. (1972). F. Neurochem. 19, 2801 .

Cocchi, D., di Giulio, A., Groppetti, A., Mantegazza, D., Müller, E. E. \& Spano, P. F. (I975). Experientia 31, $3^{84}$

Cunningham, V. J., Hay, L. \& Stoner, H. B. (1975). Biochem. F. 146, 653 .

Curzon, G., Friedel, J. \& Knott, P. J. (1973). Nature, Lond. 242, 298.

Curzon, G. \& Knott, P. J. (1974). Br. F. Pharmac. 50, 197.

Eccleston, D., Ashcroft, G. W. \& Crawford, T. B. B. (1965). F. Neurochem. 12, 493.

Etienne, P., Young, S. N., Sourkes, T. L. (1976). Nature, Lond. 262, 144 .

Fernstrom, J. D., Madras, B. K., Munro, H. N. \& Wurtman, R. J. (1974). Ciba Fdn Symp. 22, 153.

Fernstrom, J. D. \& Wurtman, R. J. (1971). Science, N.Y. 173, 149.

Green, A. R. \& Curzon, G. (1968). Nature, Lond. 220, 1095.

Hamon, M., Bourgoin, S. \& Glowinski, J. (1973). F. Neurochem. 20, 1727.

Hamon, M., Bourgoin, S., Hèry, F., Ternaux, J. P. \& Glowinski, J. (1976). Nature, Lond. 260,6 I.

Hayaishi, O. (I975). F. Biochem., Tokyo 79, I 3p. 
Kaufman, S. (1974). Ciba Fnd Symp. 22, 85.

Knott, P. J. \& Curzon, G. (1972). Nature, Lond. 239, $45^{2}$.

Liu, C.-C., Chung, C.-H. \& Lee, M.-L. (1973). Biochem. F. $135,367$.

McMenamy, R. H. \& Oncley, J. L. (1958). Y. biol. Chem. 233, 1436.

Mandell, A. J. \& Knapp, S. (1977). Fedn Proc. Fedn Am. Socs exp. Biol. 36, 2142,

Marsden, C. A. \& Curzon, G. (1976). Neuropharmacology 15, 703.

Oldendorf, W. H. (1971). Am. F. Physiol. 221 , 1629.

Pardridge, W. M. \&c Oldenforf, W. H. (1977). f. Neurochem. 28, 5.

Shields, P. J. \& Eccleston, D. (1972). $\mathcal{~}$. Neurochem. 19, 265 .

Smith, H. G. \& Lakatos, C. (1971). F. Pharm. Pharmac. 23, 180.

Tagliamonte, A., Biggio, G., Vargiu, L. \& Gessa, G. L. (1973). J. Neurochem. $20,909$.

Tagliamonte, A., Tagliamonte, P., Perez-Cruet, J. \& Gessa, G. L. (1971). Nature New Biol. 229, 125.

Yuwiler, A., Oldendorf, W. H., Geller, E. \& Braun, L. (1977). F. Neurochem. 28, 1015. 